\title{
Rapid neural representations of personally relevant faces
}

\author{
Mareike Bayer*1, Oksana Berhe ${ }^{1,2}$, Isabel Dziobek ${ }^{1}$, Tom Johnstone ${ }^{3,4}$ \\ 1 Berlin School of Mind and Brain, Department of Psychology, Humboldt-Universität zu \\ Berlin, Berlin, Germany \\ 2 Zentralinstitut für Seelische Gesundheit, 68159 Mannheim, Germany \\ 3 Centre for Integrative Neuroscience and Neurodynamics, School of Psychology and \\ Clinical Language Sciences, The University of Reading, RG6 6AH Reading, UK \\ 4 School of Health Sciences, Swinburne University of Technology, 3184 Hawthorn, \\ Australia
}

Corresponding author

Dr. Mareike Bayer

Berlin School of Mind and Brain

Department of Psychology

Humboldt-Universität zu Berlin

Unter den Linden 6, 10099 Berlin

Germany

Email: mareike.bayer@hu-berlin.de 


\section{Abstract}

The faces of those most personally relevant to us are our primary source of social information, making their timely perception a priority. Recent research indicates that gender, age and identity of faces can be decoded from EEG/MEG data within 100ms. Yet the time course and neural circuitry involved in representing the personal relevance of faces remain unknown. We applied simultaneous EEG-fMRI to examine neural responses to emotional faces of female participants' romantic partners, friends, and a stranger. Combining EEG and fMRI in cross-modal representational similarity analyses, we provide evidence that representations of personal relevance start prior to structural encoding at $100 \mathrm{~ms}$ in visual cortex, but also in prefrontal and midline regions involved in value representation, and monitoring and recall of self-relevant information. Representations related to romantic love emerged after $300 \mathrm{~ms}$. Our results add to an emerging body of research that suggests that models of face perception need to be updated to account for rapid detection of personal relevance in cortical circuitry beyond the core face processing network.

Keywords: Personal relevance, emotion, faces, EEG-fMRI, Representation 
Faces are the most important social and emotional stimuli we encounter in daily life.

They tell us whether our fellow humans are strangers, enemies, or loved ones, and how they are feeling. As face experts, we possess distinct neural pathways for processing transient (e.g. emotion) and stable (e.g. identity) aspects of faces (Bruce and Young 1986; Kanwisher 2000). Yet investigating the temporal dynamics and neural circuitry of human face perception has proved a considerable methodological challenge.

EEG and fMRI research suggests that structural face encoding and representations of identity first occur about 170ms after stimulus onset in the ventral visual stream, specifically the fusiform gyrus (Horovitz et al. 2004; Rossion and Jacques 2012). Recent more nuanced EEG \& MEG analyses have suggested that face identity, emotion, age, and gender, might be represented within 100 ms after stimulus onset (Nemrodov et al. 2016; Vida et al. 2017; Dima et al. 2018). Interestingly, a recent MEG study reported enhanced early (70-100ms) encoding of gender and identity in familiar faces (actors), with less robust encoding of gender and identity in unfamiliar faces (Dobs et al. 2019). These studies suggest that particularly relevant aspects of faces may influence processing prior to structural encoding, although the MEG and EEG analyses do not provide the spatial specificity to investigate the network underlying these effects.

The processing of familiar faces has generally been linked to more robust face detection and recognition (Ramon and Gobbini 2018), however, an additional advantage has been shown for personally relevant faces, which seem to be processed in a qualitatively different way than famous faces (Sugiura 2014; Ramon and Gobbini 2018). EEG findings suggest that the earliest effects of personal relevance of faces occur around $170 \mathrm{~ms}$ after stimulus onset, corresponding to structural encoding (Ramon and Gobbini 2018), though effects at higher-order processing stages (e.g. indexed by the P3 component) are more robust (Guerra et al. 2012). 
Consistent with these results, fMRI results have shown that the processing of personally familiar faces additionally recruits brain regions outside of the visual processing stream (the so-called extended face network), which are involved in monitoring of self-relevant information (medial prefrontal cortex), episodic memories (precuneus, anterior temporal cortex), person knowledge (temporoparietal junction; TPJ), and emotion processing (amygdala, insula) (Haxby et al. 2000; Gobbini and Haxby 2007; Natu and O’Toole 2011; Visconti Di Oleggio Castello et al. 2017).

Despite considerable progress, it remains unclear how quickly the genuine personal relevance of faces, such as those of our friends and loved ones, is decoded, and the extent to which different parts of the 'extended' face processing network are engaged at early versus late processing of personal relevance. In this study, we addressed these questions through simultaneous recording of EEG and fMRI. We presented heterosexual females in a romantic relationship with pictures of their romantic partner, a close male friend, and a male stranger, displaying fearful, happy and neutral facial expressions. The face identities were chosen in order to allow for a more fine-grained investigation of personal relevance: While partner and friend both represent personally relevant, familiar faces (as compared to a stranger), there is also a distinction between the partner and both friend and stranger, concerning feelings of romantic love. Including emotional expressions also allowed us to explore the temporal course at which emotional expressions (fear, happiness and neutral) are represented (Dima et al. 2018; Muukkonen et al. 2020).

To identify the time course and brain regions involved in representing different aspects of personally relevant faces, we combined EEG and fMRI data using representational similarity analyses (RSA; Fig 1) (Kriegeskorte 2008; Cichy and Oliva 2020). Based on previous results, we expected the involvement of the extended face processing network 
in the processing of personal relevance, possibly starting at the stage of structural encoding at $170 \mathrm{~ms}$.

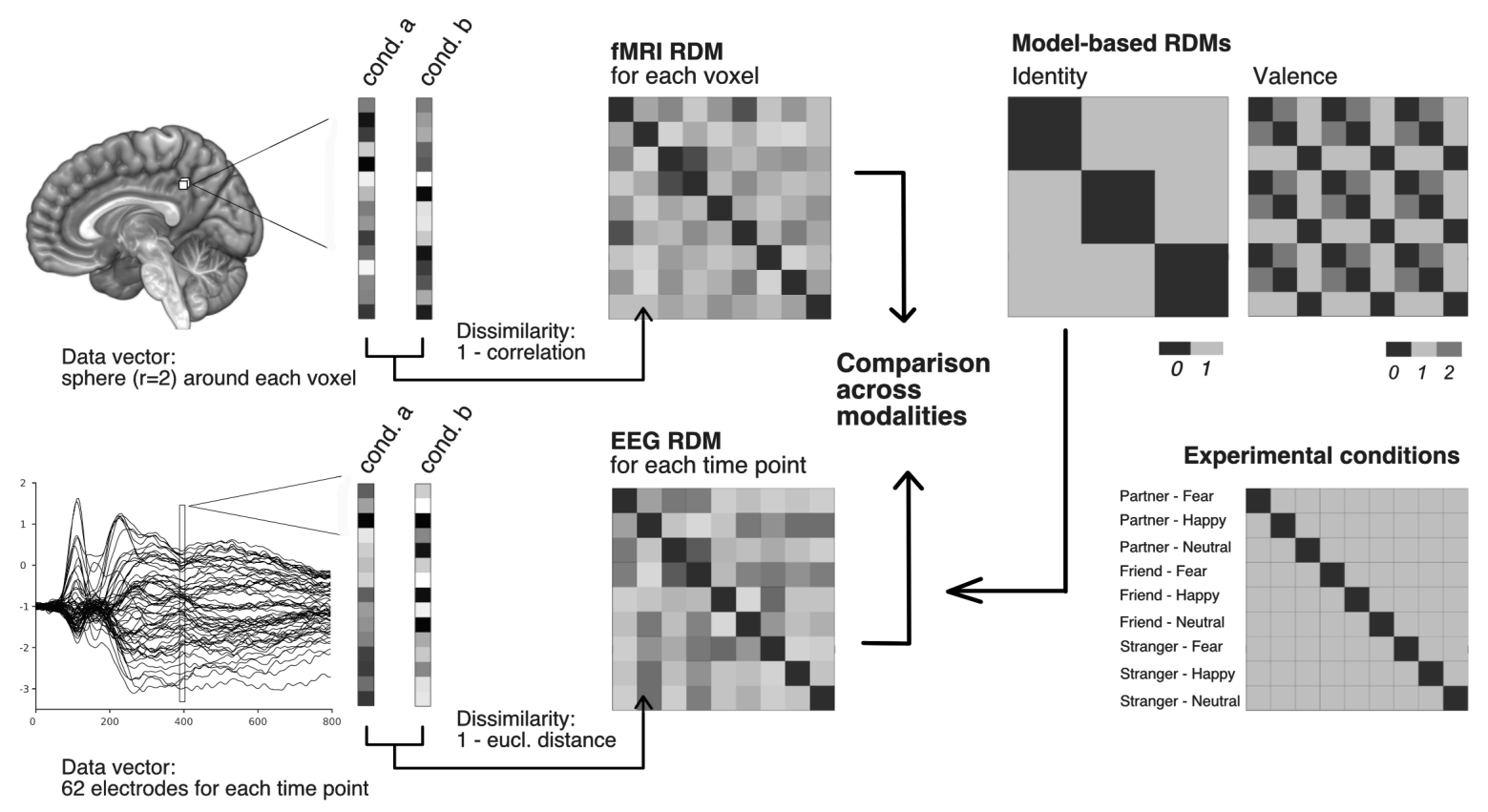

Fig. 1. Representational Similarity Analyses. Representational dissimilarity matrices (RDMs) are constructed for each voxel in the brain and for each data point in grand averaged ERPs by comparing pairwise, condition-specific activations. RDMs are symmetric with a diagonal of zeros, and their size corresponds to the number of experimental conditions, here $9 \times 9$. Model-based RDMs reflect theoretical predictions. After construction, RDMs are compared across modalities and with model-based RDMs.

\section{Materials and Methods}

The study was reviewed and approved by the University of Reading research ethics committee. All participants provided informed consent before taking part in the study.

\section{Participants}

Data were collected from 22 female participants (mean age $=19.8$ years, $s d=0.9$ ). For four participants, no sufficient EEG data was available (for 2 subjects only 32 channel EEG was recorded, one participant was wearing a hair weave, one participant's data was excluded due to excessive artefacts). All participants were in a heterosexual romantic 
relationship at the time of data collection (mean duration $=20.0$ months, $\mathrm{sd}=14.6$;

friendship: mean duration $=35.9$ months, $s d=26.5$ ). Participants received a mean score of 105.9 points $(s d=10.7$ ) of 135 points on the Passionate Love Scale (Hatfield and Sprecher 1986). They reported high contentment both with their relationship (mean = 8.8/10, sd $=1.3$ ) and their friendship (mean $=8.3, \mathrm{sd}=1.3$ ). All participants had normal or corrected-to-normal vision; 20 participants were right-handed. Participants were recruited through the Undergraduate Research Panel and Internet ads; they received course credit or $£ 25$ for participation.

\section{Stimuli}

Stimuli consisted of portraits of the participant's boyfriend, a male close friend, and a male stranger, displaying fearful, happy, and neutral facial expressions ( 3 x 3 design). All stimuli were obtained by taking screen shots during a Skype session prior to the experimental session; all participants were presented with the same stranger.

After the main experiment, participants completed ratings of stimulus valence and arousal, as well as on attractiveness (neutral expression) using 7-point Likert scales. Ratings are reported in Table 1.

Table 1. Rating values (means and standard deviations) of experimental stimuli

\begin{tabular}{|c|c|c|c|c|c|c|c|}
\hline & \multirow[t]{2}{*}{$\begin{array}{c}\text { Attractiveness } \\
(1-7)\end{array}$} & \multicolumn{3}{|c|}{$\begin{array}{c}\text { Valence } \\
(-3-3)\end{array}$} & \multicolumn{3}{|c|}{$\begin{array}{c}\text { Arousal } \\
(1-7)\end{array}$} \\
\hline & & Fearful & Happy & Neutral & Fearful & Happy & Neutral \\
\hline Partner & $6.1(1.1)$ & $-0.4(1.5)$ & $2.3(0.7)$ & $0.0(1.8)$ & $3.2(1.7)$ & $5.4(1.5)$ & $3.7(1.8)$ \\
\hline Friend & $4.1(1.4)$ & $-1.1(1.1)$ & $1.6(0.8)$ & $-0.6(1.1)$ & $2.1(1.6)$ & 2.9 (1.9) & $2.5(1.6)$ \\
\hline Stranger & $4.1(1.2)$ & $-2.0(1.1)$ & $0.5(1.1)$ & $-0.6(1.2)$ & $1.6(1.1)$ & $3.2(2.0)$ & $2.5(1.6)$ \\
\hline
\end{tabular}




\section{Procedure}

After receiving information about the study, participants provided informed consent and were fitted with the EEG cap. Inside the scanner, participants performed a passive face-viewing task. Face stimuli were presented for 1s. Every face stimulus was presented 40 times, resulting in 360 experimental trials. Additionally, 40 1-back trials were presented to ensure participant's attention to the faces. In these trials, participants had to indicate whether a face presented after a question mark was identical in identity and emotion to the one presented before the question mark. Stimuli were presented in 10 blocks. The stimulus sequence and the jittering of the inter-trial interval was determined using fMRI simulator (Rorden 2011) (mean ITI $=3000 \mathrm{~ms}, \mathrm{~min}=2500 \mathrm{~ms}$, exponential function). A central fixation cross was presented during the ITI. Stimuli were presented on a Nordic Neuro Labs goggle system at $60 \mathrm{~Hz}$ on an $800 \times 600$ pixel screen using EPrime software (Psychology Software Tools, Inc.).

\section{Data acquisition and pre-processing}

fMRI

\section{Acquisition}

Data were collected on a 3T Siemens Trio MRI scanner using the standard 12 channel head coil. Functional images were acquired with a T2*-weighted gradient echo EPI sequence (40 interleaved axial slices, phase encoding P to A, 2.5 x $2.5 \mathrm{~mm}$ voxels, slice thickness $=2.5 \mathrm{~mm}$, interslice gap $=0.25 \mathrm{~mm}, 100 \times 100$ in-plane matrix, $\mathrm{TR}=2500 \mathrm{~ms}$, TE = 30 ms, Flip Angle: 90 . A high-resolution T1-weighted whole-brain structural image was acquired using an MPRAGE sequence $(1 \mathrm{~mm}$ isotropic voxels, FOV $=160 \times 256$ x 256 mm, Flip Angle: 9²).

Preprocessing and GLM 
Data were processed with FSL 5.0 (http://www.fmrib.ox.ac.uk/fsl). Brain extraction was performed using the BET (Smith 2002). Data were denoised (Marchenko-Pastur Principal Component Analyses), motion corrected (Jenkinson et al. 2002), spatially smoothed using a 5 mm FWHM Gaussian kernel (Smith and Brady 1997), and grandmean intensity normalized. Motion artefacts were removed with ICA-AROMA (Pruim et al. 2015) and data were high-pass filtered (100 s). Single subject 4D data were registered to the subject's structural image using BBR (Jenkinson and Smith 2001). Registration of the individual structural image to the 2mm MNI 152 template was performed using ANTS (Avants et al. 2009). Finally, transformations were combined for the registration of the functional data to standard space. A first level GLM was applied with regressors for emotion by identity conditions ( 9 regressors) and for 1-back trials, created by convolving the temporal profile of each experimental condition with the FSL double gamma haemodynamic response function. Nuisance regressors without convolution were included to model breaks between blocks and artefacts (framewise displacement $>1 \mathrm{~mm})$.

RDMs

Representational dissimilarity matrices (RDMs; Kriegeskorte 2008; Cichy et al. 2016)were constructed for each voxel in the brain, separately for each subject, based on z-statistic images of each of the 9 experimental conditions included in the mixed-effects GLM. For each voxel and condition, we created a vector (based on a sphere around each voxel, radius $=2$ voxels). We quantified the dissimilarity between each pair of experimental conditions as 1 - Pearson's R of the two corresponding vectors. This resulted in a $9 \times 9$ RDM for each voxel and each participant. For unimodal second-level fMRI analyses and results, see supplementary material. 


\section{EEG}

Data acquisition

Continuous EEG data were collected from $64 \mathrm{Ag}$-AgCl electrodes simultaneously to fMRI acquisition (BrainProducts system); the electrocardiogram (ECG) was recorded from an electrode placed left of the spinal column. The sampling rate was $5000 \mathrm{~Hz}$; data were referenced online to electrode $\mathrm{FCz}$ with electrode $\mathrm{AFz}$ as ground. Electrode impedances were kept below $20 \mathrm{k} \Omega$. A sync box (BrainProducts) synchronised the MRI and EEG computer clocks.

Preprocessing

Offline, MR gradient artefacts were removed from continuous, baseline corrected data (using the whole artefact for baseline correction) with a sliding window of 21 artefacts (Allen et al. 2000). Data were low-pass filtered using a FIR filter (70 Hz) and downsampled to $250 \mathrm{~Hz}$. Ballistocardiographic artefacts were identified using the ECG with a semiautomatic template matching procedure and corrected using a template subtraction approach (sliding window of 15 pulse intervals). A restricted Infomax ICA (Bell and Sejnowski 1995) was used to remove eye blinks, eye movements and residual ballistocardiographic artefacts. Data was re-referenced to average reference and segmented into epochs from -100 ms to $800 \mathrm{~ms}$ relative to stimulus onset, and baselinecorrected using a $100 \mathrm{~ms}$ pre-stimulus baseline. Trials with activity exceeding $\pm 100 \mu \mathrm{V}$ or voltage steps larger than $100 \mu \mathrm{V}$ were excluded from analyses ( $0.6 \%$ of trials). Data were averaged per participant and experimental condition.

\section{RDMs}

RDM matrices were constructed using grand-averaged waveforms. For each time point from stimulus onset to $800 \mathrm{~ms}$ after stimulus onset, the distance between pairs of experimental conditions was quantified as their Euclidean distance across all 62 scalp 
electrodes. Euclidean distance was used in order to account for amplitude differences, which convey essential information in event-related potentials. Analyses resulted in a 9 x 9 RDM for each time point.

In order to constrain target EEG RDMs in the cross-modal analyses, we derived a measure of internal structure of the EEG RDM at each time point, in order to identify time points with potentially high representational content. For each time point's RDM, we computed Euclidean distances of each RDM cell value to the arithmetic mean of all cell values in that RDM. As a result, EEG time points with pronounced differences between specific condition pairs relative to the mean difference between all pairs receive high values, whereas relatively smaller differences result in low values. For these calculations, we used one half of the (symmetrical) RDM, excluding the diagonal zeros. Time points of interest (peaks in EEG RDM structure) were identified at $52 \mathrm{ms,}$ 108 ms, 204 ms, 308 ms, 428 ms, and 660 ms (see Fig. 2).

\section{Data analyses}

Model-based EEG regression analyses

To characterize the information space represented by EEG-RDMs, we performed linear regression analyses on EEG RDMs at the time points of interest using model RDMs as explanatory variables. In other words, we wanted to determine how experimental factors were represented throughout the EEG time line. Model RDMs for Identity were created based on the assumption of high similarity within and low similarity across identity (coded as 0 and 1, respectively). We also computed model RDMs for face familiarity (Partner/Friend vs. Stranger) and romantic love (Partner vs. Friend/Stranger). Emotion conditions were represented as emotional valence (distance between happy/fearful vs. neutral = 1, distance happy vs. fearful = 2) and arousal 
(distance happy $/$ fearful vs. neutral $=1$, distance happy vs. fearful $=0$ ). For each time point of interest in the EEG, we entered RDMs for Identity, Familiarity, Love, Valence and Arousal as explanatory variables in the regression analysis.

\section{EEG-fMRI fusion}

After RDMs had been constructed for each voxel in the brain, EEG RDMs at time points of interest (as identified by our measure of internal structure) were used as target RDMs in single subject cross-modal searchlight analyses (using Pearson's R), in order to identify brain regions with corresponding fMRI-derived representations. The resulting single subject RSA brain maps were entered into whole-brain second level analyses using permutation tests (with an MNI gray matter mask with tissue priors thresholded at 0.3 and variance smoothing of $2 \mathrm{~mm}$ ). Threshold-free cluster enhancement was used to ensure a corrected Familywise Error (FWE) of <.05. Using permutation tests for second level analyses mitigates against bias in the first level RSA, since the permutation procedure produces a null distribution that incorporates the bias (hence if lower level estimates are positively biased, the null distribution is as well, and hence the criterion for significance at a given alpha is adjusted upwards).

EEG-fMRI regression analyses including visual feature models In order to control for possible effects of low-level visual features on RSA results, we constructed RDMs for each stimulus based on a model of cortical responses of neurons in the primary visual cortex (HMAX; Serre et al. 2007), more specifically on the output of the second layer (C1) of the model. RDMs for each participant's individual stimulus set were calculated using Pearson's correlations. These RDMs were included as covariates 
in posthoc cross-modal RSA regression analyses to assess whether results remained significant when accounting for representations of low-level stimulus features.

RSA analyses were performed using the CoSMoMVPA toolbox (Oosterhof et al. 2016) and the toolbox for representational similarity analyses (Nili et al. 2014) on Matlab R2017b.

\section{Results}

Peaks in EEG RDM structure were evident at $52 \mathrm{~ms}, 108 \mathrm{~ms}, 204 \mathrm{~ms}, 308 \mathrm{~ms}, 428 \mathrm{~ms}$, and 660 ms. Accordingly, these RDMs were used as searchlight RDMs in the whole-brain analyses on single-subject fMRI data (Fig. 2). Model-based EEG regression analyses showed correspondence of EEG representations with RDMs of Familiarity at time points from 108 ms onward, and additional correspondence to the Love-RDM from 300 ms. Interestingly, Valence and Arousal did not show significant correspondence at any of the time points (see Table 2).

In combined EEF-fMRI analyses, there were no brain regions with significantly correlated spatial RDMs for the EEG RDM at 52 ms after stimulus onset. EEG representations at $108 \mathrm{~ms}$ showed correspondence to BOLD representations in the visual cortex (intracalcarine cortex and lingual gyrus), but also in the extended face network, including the the ventromedial PFC, precuneus, posterior cingulate cortex and TPJ . The EEG RDM at this time point mainly represented effects of familiarity (Stranger vs. Partner and Friend), as apparent from linear regression analyses of the EEG RDM (see Table 2). The same was true for the EEG RDM at 204 ms, with more widespread corresponding EEG-fMRI representations that additionally included the ACC, fusiform gyrus, amygdala, insula and N. accumbens. At 308 ms and 428 ms, EEG RDMs showed 
highest correspondence to model-based RDMs of Love (that is, Partner vs. Friend and Stranger). In EEG-fMRI, corresponding representations at 308 ms were more focal and most evident in the precuneus, posterior cingulate and superior frontal gyrus. Finally, EEG-fMRI representations at $428 \mathrm{~ms}$ and $660 \mathrm{~ms}$ after stimulus onset were again more extended, with additional significant representations in the middle temporal gyrus, TPJ, in the inferior and superior frontal gyri, as well as in regions of the midbrain. In linear regression analyses, the EEG RDM showed correspondence to both the Familiarity and the Love model RDMs.

Results of RSA regression analyses which included an RDM model of cortical responses in the visual cortex (HMAX) confirmed our previous analyses, showing that significant representations were not determined by lower-level stimulus features. See Table 3 for a complete list of brain regions and the results of RSA HMAX regressor analyses.

Table 2. Results of linear regression models on EEG-RDMs and conceptual model RDMs. Standardized parameter estimates and $\mathrm{p}$-values are given for each conceptual model $\mathrm{RDM}$, as well as model adjusted $\mathrm{R}^{2}$.

\begin{tabular}{lcccccc}
\hline & $\mathbf{5 2} \mathbf{~ m s}$ & $\mathbf{1 0 8} \mathbf{~ m s}$ & $\mathbf{2 0 4} \mathbf{~ m s}$ & $\mathbf{3 0 8} \mathbf{~ m s}$ & $\mathbf{4 2 8} \mathbf{~ m s}$ & $\mathbf{6 6 0 ~} \mathbf{~ m s}$ \\
Identity & -0.31 & -0.25 & -0.27 & -0.50 & -0.23 & -0.23 \\
& $p=.287$ & $p=.371$ & $p=.293$ & $p=.052$ & $p=.250$ & $p=.309$ \\
Familiarity & -0.01 & 0.63 & 0.76 & 0.53 & 0.55 & 0.76 \\
& $p=.957$ & $p=.007$ & $p<.001$ & $p=.011$ & $p=.002$ & $p<.001$ \\
Love & 0.3464 & 0.30 & 0.29 & 0.80 & 0.79 & 0.50 \\
& $p=.145$ & $p=.177$ & $p=.149$ & $p<.001$ & $p<.001$ & $p=.008$ \\
Valence & -0.29 & 0.05 & 0.13 & -0.09 & 0.01 & 0.06 \\
& $p=.101$ & $p=.749$ & $p=.383$ & $p=.549$ & $p=.918$ & $p=.635$ \\
Arousal & 0.17 & -0.08 & -0.01 & 0.07 & 0.00 & -0.07 \\
& $p=.325$ & $p=.602$ & $p=.968$ & $p=.608$ & $p=1$ & $p=.587$ \\
Adjusted $\mathbf{R}^{2}$ & 0.063 & 0.172 & 0.311 & 0.324 & 0.560 & 0.471 \\
& & & & & & \\
\hline
\end{tabular}




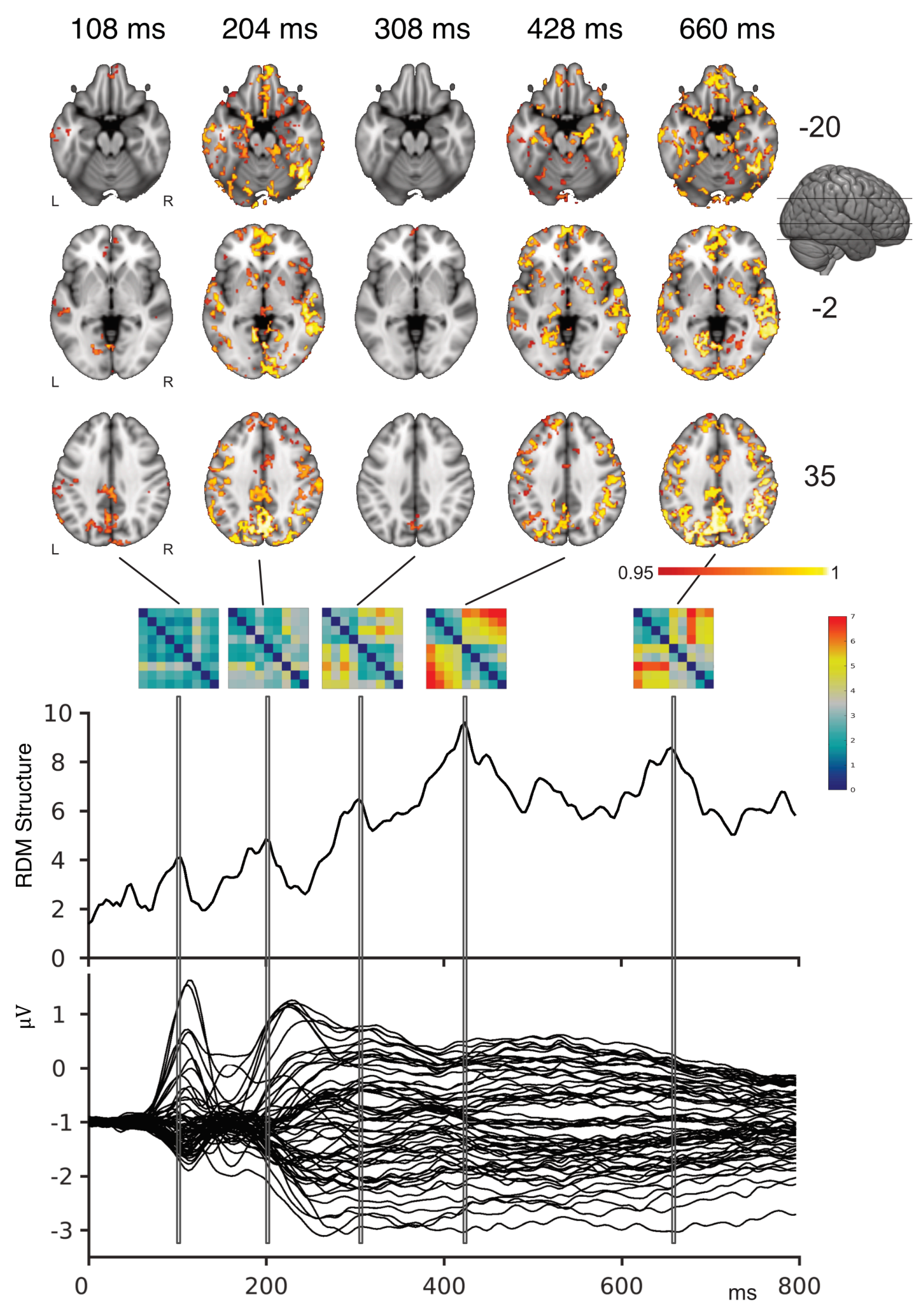


Fig. 2: Combined EEG-fMRI representations. Brain regions in which fMRI RDMs corresponded to representations in the EEG at the corresponding time points. RDMs show EEG representations used as target RDMs in the combined EEG-fMRI analyses, selected on the basis of peaks in the RDM structure (top graph). Each line in the lower graph represents the mean time series for one electrode.

Table 3. Summary of brain activations. Activations are whole-brain corrected using permutation tests and threshold-free cluster enhancement ( FWE < 0.05). Cluster locations are reported based on t-statistics after thresholding with the binarised result of the TFCE. Only the 10 largest clusters per condition are reported. Cluster size reported in $2 \times 2 \times 2 \mathrm{~mm}$ voxels. Right-most column indicates clusters remaining significant after covarying with HMAX regressors $\left(\mathrm{p}<0.01\left({ }^{*}\right)\right.$ and $\mathrm{p}<0.001\left(^{* *}\right)$, uncorrected).

\begin{tabular}{|c|c|c|c|c|c|c|}
\hline \multirow[t]{2}{*}{ Condition } & \multirow[t]{2}{*}{ Brain regions } & \multicolumn{3}{|c|}{ Local maxima $(\mathrm{mm})$} & \multirow{2}{*}{$\begin{array}{l}\text { Cluster size } \\
\quad \text { (voxels) }\end{array}$} & \multirow{2}{*}{$\begin{array}{l}\text { Model } \\
\text { incl. } \\
\text { HMAX }\end{array}$} \\
\hline & & $x$ & $y$ & $z$ & & \\
\hline \multirow[t]{10}{*}{$108 \mathrm{~ms}$} & $\begin{array}{l}\text { Lingual gyrus, intracalcarine cortex, } \\
\text { precuneous cortex, cuneal cortex }\end{array}$ & -2 & -68 & 2 & 620 & ** \\
\hline & $\begin{array}{l}\text { L planum temporale, middle temporal } \\
\text { gyrus, superior temporal gyrus, } \\
\text { supramarginal gyrus }\end{array}$ & -62 & -32 & 12 & 449 & $* *$ \\
\hline & Precuneus cortex & 0 & -62 & 38 & 200 & * \\
\hline & $\begin{array}{l}\text { L superior parietal lobule, lateral occipital } \\
\text { cortex }\end{array}$ & -26 & -58 & 54 & 170 & * \\
\hline & R lateral occipital cortex & 36 & -72 & 54 & 156 & $* *$ \\
\hline & Posterior cingulate cortex & -6 & -26 & 34 & 132 & * \\
\hline & $\mathrm{L}$ middle temporal gyrus & -58 & -8 & -14 & 125 & * \\
\hline & $\begin{array}{l}\text { L lateral occipital cortex, angular gyrus, } \\
\text { supramarginal gyrus }\end{array}$ & -60 & -62 & 32 & 81 & ** \\
\hline & Ventromedial cortex, frontal pole & 2 & 52 & -22 & 79 & ** \\
\hline & L lateral occipital cortex & -52 & -68 & 22 & 79 & $* *$ \\
\hline \multirow[t]{3}{*}{$204 \mathrm{~ms}$} & $\begin{array}{l}\text { Lingual gyrus, Precuneous cortex, } \\
\text { posterior cingulate cortex }\end{array}$ & 0 & -68 & 2 & 2890 & $* *$ \\
\hline & $\begin{array}{l}\mathrm{R} \text { inferior temporal gyrus, lateral occipital } \\
\text { cortex, superior temporal sulcus, middle } \\
\text { temporal gyrus, fusiform cortex, } \\
\text { cerebellum }\end{array}$ & 48 & -58 & -16 & 2442 & $* *$ \\
\hline & $\begin{array}{l}\text { Ventromedial prefrontal cortex, frontal } \\
\text { pole, anterior cingulate cortex }\end{array}$ & 0 & 48 & -14 & 1111 & ** \\
\hline
\end{tabular}




\begin{tabular}{|c|c|c|c|c|c|c|}
\hline & $\begin{array}{l}\text { Medial forebrain, hypothalamus, L } \\
\text { amygdala, N. accumbens, BNST }\end{array}$ & -2 & -2 & -12 & 619 & ** \\
\hline & $\begin{array}{l}\mathrm{L} \text { middle temporal gyrus, superior } \\
\text { temporal gyrus, planum temporale }\end{array}$ & -58 & -10 & -10 & 528 & ** \\
\hline & $\begin{array}{l}\text { L lateral occipital cortex, supramarginal } \\
\text { gyrus, postcentral gyrus, precentral gyrus }\end{array}$ & -56 & -64 & 36 & 336 & * \\
\hline & R precentral gyrus, middle frontal gyrus & 44 & -6 & 46 & 335 & ** \\
\hline & $\begin{array}{l}\mathrm{R} \text { orbitofrontal cortex, insular cortex, } \\
\text { frontal pole }\end{array}$ & 32 & 26 & -18 & 256 & ** \\
\hline & Posterior cingulate gyrus, precentral gyrus & -4 & -38 & 34 & 207 & ** \\
\hline & Cerebellum, fusiform gyrus & -14 & -80 & -20 & 203 & * \\
\hline \multirow[t]{10}{*}{$308 \mathrm{~ms}$} & Precuneous cortex & 2 & -76 & 42 & 119 & * \\
\hline & $\mathrm{R}$ superior frontal gyrus & 10 & 14 & 68 & 59 & ** \\
\hline & Frontal pole & -8 & 60 & -8 & 47 & ** \\
\hline & Precuneous cortex, intracalcarine cortex & -6 & -64 & 18 & 28 & * \\
\hline & Posterior cingulate gyrus & -2 & -44 & 10 & 20 & * \\
\hline & R occipital pole & 16 & -90 & 8 & 19 & * \\
\hline & Lingual gyrus & -4 & -64 & 2 & 14 & ** \\
\hline & $\mathrm{R}$ precuneous cortex & 16 & -68 & 46 & 7 & * \\
\hline & $L$ temporal fusiform gyrus & -34 & -20 & -30 & 6 & * \\
\hline & L postcentral gyrus & -10 & -40 & 74 & 4 & ** \\
\hline \multirow[t]{10}{*}{$428 \mathrm{~ms}$} & R middle temporal gyrus, angular gyrus & 66 & -28 & -2 & 233 & $* *$ \\
\hline & Frontal pole & -4 & 64 & 0 & 228 & ** \\
\hline & L precentral gyrus, postcentral gyrus & -24 & -26 & 62 & 227 & ** \\
\hline & R superior frontal gyrus & 10 & 16 & 68 & 214 & ** \\
\hline & $\begin{array}{l}\text { L central opercular cortex, precentral } \\
\text { gyrus, postcentral gyrus, planum polare }\end{array}$ & -50 & 4 & 6 & 210 & ** \\
\hline & $\begin{array}{l}\mathrm{R} \text { angular gyrus, lateral occipital cortex, } \\
\text { precuneous cortex }\end{array}$ & 40 & -56 & 34 & 184 & ** \\
\hline & $\begin{array}{l}L \text { inferior frontal gyrus, orbitofrontal cortex, } \\
\text { frontal operculum }\end{array}$ & -54 & 14 & 10 & 180 & * \\
\hline & $\mathrm{L}$ middle frontal gyrus & -44 & 6 & 44 & 179 & ** \\
\hline & $\begin{array}{l}\text { R middle frontal gyrus, inferior frontal } \\
\text { gyurs }\end{array}$ & 52 & 36 & 28 & 176 & ** \\
\hline & $\begin{array}{l}\text { Precuneus cortex, posterior cingulate } \\
\text { cortex }\end{array}$ & -10 & -54 & 46 & 167 & * \\
\hline
\end{tabular}




\begin{tabular}{|c|c|c|c|c|c|c|}
\hline \multirow[t]{10}{*}{$660 \mathrm{~ms}$} & $\begin{array}{l}\text { Lateral occipital cortex, Intracalcarine } \\
\text { cortex, precuneous cortex, cuneal cortex, } \\
\text { posterior cingulate cortex, lingual gyrus, } \\
\text { middle frontal gyrus, superior frontal gyrus }\end{array}$ & 54 & -68 & 24 & 5570 & ** \\
\hline & $\begin{array}{l}\text { R superior temporal gyrus, middle } \\
\text { temporal gyrus, inferior temporal gyrus, } \\
\text { planum temporale, supramarginal gyrus, } \\
\text { cerebellum }\end{array}$ & 60 & -8 & -4 & 1339 & ** \\
\hline & $\begin{array}{l}\text { Frontal pole, paracingulate gyrus, frontal } \\
\text { medial cortex, subcallosal cortex, superior } \\
\text { frontal gyrus }\end{array}$ & -24 & 66 & 10 & 1150 & ** \\
\hline & $\begin{array}{l}\mathrm{R} \text { middle frontal gyrus, frontal pole, inferior } \\
\text { frontal gyrus, frontal operculum, frontal } \\
\text { orbital cortex }\end{array}$ & 48 & 34 & 24 & 757 & ** \\
\hline & Lingual gyrus, occipital pole & 8 & -88 & -14 & 660 & ** \\
\hline & Cerebellum, fusiform cortex & -36 & -52 & -28 & 386 & ** \\
\hline & $\begin{array}{l}\text { L postcentral gyrus, precentral gyrus, } \\
\text { central operculum }\end{array}$ & -50 & -6 & 18 & 286 & * \\
\hline & L superior frontal gyrus & -14 & 18 & 52 & 284 & * \\
\hline & L precentral gyrus, precentral gyrus, & -12 & -34 & 64 & 224 & ** \\
\hline & $\mathrm{R}$ middle frontal gyrus & 50 & 20 & 38 & 223 & ** \\
\hline
\end{tabular}

\section{Discussion}

The faces of those closest to us are arguably the most personally relevant social stimuli we encounter and thus might receive processing priority. In this study, we investigated the time course of neural processing of the faces of romantic partners and friends using simultaneous EEG-fMRI and representational similarity analyses. Our results point to the strong and rapid impact of personal relevance on face processing, with shared representations between EEG and fMRI spatial activation patterns in multiple cortical regions starting as early as $100 \mathrm{~ms}$ after stimulus onset. 
The combination of EEG and fMRI data using RSA allows for the time-resolved analyses of representations within the fMRI. These analyses suggest fast modulation of neural processing across a far more widespread collection of cortical regions than predicted. EEG representations which corresponded to personal familiarity were first apparent as early as $100 \mathrm{~ms}$ after stimulus onset. Corresponding fMRI representations were found not only in the visual cortex, but also in the ventromedial and medial prefrontal cortex, regions involved in the monitoring of self-relevant information and value encoding (Northoff et al. 2006; Berridge and Kringelbach 2015). Further early representations were observed in regions linked to multimodal sensory integration and theory of mind (TPJ) (Patel et al. 2019), and episodic and autobiographical memory (posterior cingulate cortex) (Leech and Sharp 2014). The fast and widespread activation of brain areas involved in social cognition and reward encoding serves to highlight the prioritization of genuinely personally relevant information: In contrast to our findings, a previous MEG study using famous faces instead of personally relevant faces reported decoding of face familiarity only after $400 \mathrm{~ms}$ (Dobs et al. 2019). However, that study reported increased early representations of gender and identity for familiar compared to unfamiliar faces with latencies of 60 to $100 \mathrm{~ms}$. Based on the timeline of their results, the authors speculated that these activations might be based on selective bottom-up amplification of sensory representations. In contrast, although starting somewhat later, our results show that at $100 \mathrm{~ms}$ after stimulus onset, corresponding fMRI representations were evident outside of visual areas, including the PFC.

The EEG-fMRI RSA analyses suggest that personal relevance of faces can be extracted prior to full structural face encoding. These results add to recent EEG and MEG research suggesting that various aspects of faces including identity, emotion, age, and gender, might be represented within $100 \mathrm{~ms}$ after stimulus onset (Nemrodov et al. 2016; Vida et 
al. 2017; Dima et al. 2018). Our results indicate the rapid involvement of both visual and frontal/midline brain regions. Based on this circuitry, we suggest that these effects might rely on associative learning of visual stimulus features and the reward value of personally relevant faces. This explanation is consistent with reports of fast activations of the PFC in affective associative learning of faces at latencies starting as early as 50 $80 \mathrm{~ms}$ after stimulus onset (Steinberg et al. 2013). Therefore, future models of face perception should consider the influence of face-unspecific mechanisms on face perception, like reward-related processes, which seem to play an important role in processing real-life faces.

At $200 \mathrm{~ms}$, shortly after the stage of structural face encoding, representations of familiarity additionally included the fusiform gyrus, but also amygdala, insular cortex and $\mathrm{N}$. accumbens. This time course of representations of face relevance suggests that the response of subcortical relevance- and reward related structures like the amygdala and the N. accumbens might rely on the output of structural face encoding at around 170 ms after stimulus onset.

Finally, at the stage of higher-order processing from approximately $400 \mathrm{~ms}$ after stimulus onset, representations of familiarity and love were identified in all regions of the core and extended face processing network, including amygdala, insular and orbitofrontal cortex (Gobbini and Haxby 2007), but also in putamen, cerebellum and regions of the brain stem. Taken together, our results suggest that early processing mainly reflects the amplification of familiarity; whereas effects specific for romantic love only emerge at a higher-order processing stage. However, it is important to keep in mind that the terms 'familiarity' and 'love' in this case are not exclusively related to Friend and Partner, respectively, but reflect different aspects of close relationships (Hendrick and Hendrick 1986): Both Partner and Friend are of high personal relevance; 
and one can feel both friendship love towards a close friend as well as friendship towards one's partner. Thus, romantic love in our study refers to representations that are unique to a romantic relationship, over and above a close friendship.

In this study, although effects of valence consistent with previous research were present in unimodal fMRI analyses (see supplementary material), EEG regression analyses showed that the representational structure of the EEG signal exclusively reflected conceptual representations of Familiarity and Love. This should not come as a surprise. Being able to rapidly respond to those closest to us is a fundamental ability present from early infancy (Pascalis et al. 1995). It also suggests that emotional expressions are not processed in a rigid, automatic way, but that the representational space of brain responses reflects context-specific relevance of social stimuli.

Some limitations to our study design were imposed by our multimodal setup, limiting the number of trials and stimulus conditions used within a reasonable length session. A larger number of stimulus conditions would provide larger RDMs, able to capture more detailed representational structure. In addition, cross-validation of RDMs was not performed, as BOLD response estimates based on half the data were not reliable. However, this problem is mitigated by the multimodal analysis approach; crucially, we do not infer directly on unimodal RDMs, but infer on the similarity between fMRI RDMs and EEG RDMs, and EEG RDMs and model RDMs. Should fMRI or EEG RDMs have spurious, artifact or noise-driven structure, one would not expect robust relationships with model RDMs or RDMs from the other modality.

Understanding the time course and neural circuitry of processing individually relevant faces might be of special importance in clinical conditions, where processing of emotional or social information is specifically disrupted. Autism spectrum conditions 
(ASC) are an example in which atypical face processing might reflect altered personal relevance attached to strangers, rather than a dysfunction of the neural face processing architecture (Pierce 2004), with potential consequences for the design of targeted interventions.

What is clear is that in basic social neuroscience, as well as in clinical research, there are compelling reasons to study responses to individualised social stimuli. Such studies might yield valuable insights into how the brain engages circuitry beyond that typically considered in models of face perception, in order to prioritise processing of genuinely personally relevant faces. After all, by far the most pervasive social stimuli we encounter in our daily lives, the ones our brains are most attuned to, are our close friends and loved ones.

Acknowledgements

M.B. and T.J. were supported by the Berlin School of Mind and Brain (HumboldtUniversität zu Berlin) and the Erasmus program. The authors thank Michael Lindner and Catriona Scrivener for assistance with data collection and Luca Brivio for assistance with data analysis.

\section{Author contributions}

M.B., T.J. and I.D. designed the experiment, M.B. and T.J. collected data, conducted analyses and drafted the manuscript, M.B. and O.B. analysed EEG data. All authors provided feedback and revised the manuscript.

The authors declare no conflict of interest. 
Acknowledgements:

M.B. and T.J. were supported by the Berlin School of Mind and Brain (Humboldt-

Universität zu Berlin) and the Erasmus program. The authors thank Michael Lindner and

Catriona Scrivener for assistance with data collection and Luca Brivio for assistance with data analysis.

\section{References}

Allen PJ, Josephs O, Turner R. 2000. A Method for Removing Imaging Artifact from Continuous EEG Recorded during Functional MRI. NeuroImage. 12(2):230-239. doi:10.1006/nimg.2000.0599.

Avants BB, Tustison N, Song G. 2009. Advanced Normalization Tools (ANTS). Insight Journal. doi:http://hdl.handle.net/10380/3113.

Bell AJ, Sejnowski TJ. 1995. An information-maximization approach to blind separation and blind deconvolution. Neural computation. 7(6):1129-59.

Berridge KC, Kringelbach ML. 2015. Pleasure Systems in the Brain. Neuron. doi:10.1016/j.neuron.2015.02.018.

Bruce V, Young A. 1986. Understanding face recognition. British Journal of Psychology. doi:10.1111/j.2044-8295.1986.tb02199.x.

Cichy RM, Oliva A. 2020. A M/EEG-fMRI Fusion Primer: Resolving Human Brain Responses in Space and Time. Neuron. 107(5):772-781.

doi:10.1016/j.neuron.2020.07.001.

Cichy RM, Pantazis D, Oliva A. 2016. Similarity-Based Fusion of MEG and fMRI Reveals Spatio-Temporal Dynamics in Human Cortex During Visual Object Recognition. Cereb Cortex. 26(8):3563-3579. doi:10.1093/cercor/bhw135.

Dima DC, Perry G, Messaritaki E, Zhang J, Singh KD. 2018. Spatiotemporal dynamics in human visual cortex rapidly encode the emotional content of faces. Human Brain Mapping. doi:10.1002/hbm.24226.

Dobs K, Isik L, Pantazis D, Kanwisher N. 2019. How face perception unfolds over time. Nature communications. doi:10.1038/s41467-019-09239-1.

Gobbini MI, Haxby J V. 2007. Neural systems for recognition of familiar faces. 45(1):32- 
41. doi:10.1016/j.neuropsychologia.2006.04.015.

Guerra P, Vico C, Campagnoli R, Sánchez A, Anllo-vento L, Vila J. 2012. Affective processing of loved familiar faces : Integrating central and peripheral electrophysiological measures. International Journal of Psychophysiology. 85(1):79-87. doi:10.1016/j.ijpsycho.2011.06.004.

Hatfield E, Sprecher S. 1986. Measuring passionate love in intimate relationships. Journal of Adolescence. 9(4):383-410. doi:10.1016/S0140-1971(86)80043-4.

Haxby J V., Hoffman EA, Gobbini MI. 2000. The distributed human neural system for face perception. Trends in Cognitive Sciences. 4(6):223-233. doi:10.1016/S13646613(00)01482-0.

Hendrick C, Hendrick S. 1986. A Theory and Method of Love. Journal of Personality and Social Psychology. doi:10.1037/0022-3514.50.2.392.

Horovitz SG, Rossion B, Skudlarski P, Gore JC. 2004. Parametric design and correlational analyses help integrating fMRI and electrophysiological data during face processing. NeuroImage. 22(4):1587-1595. doi:10.1016/J.NEUROIMAGE.2004.04.018.

Jenkinson M, Bannister P, Brady M, Smith S. 2002. Improved optimization for the robust and accurate linear registration and motion correction of brain images. NeuroImage. 17(2):825-41.

Jenkinson M, Smith S. 2001. A global optimisation method for robust affine registration of brain images. Medical Image Analysis. doi:10.1016/S1361-8415(01)00036-6. Kanwisher N. 2000. Domain specificity in face perception. Nature Neuroscience. doi:10.1038/77664.

Kriegeskorte N. 2008. Representational similarity analysis - connecting the branches of systems neuroscience. Frontiers in Systems Neuroscience. doi:10.3389/neuro.06.004.2008.

Leech R, Sharp DJ. 2014. The role of the posterior cingulate cortex in cognition and disease. Brain. 137(1):12-32. doi:10.1093/brain/awt162.

Muukkonen I, Ölander K, Numminen J, Salmela VR. 2020. Spatio-temporal dynamics of face perception. NeuroImage. 209:116531. doi:10.1016/j.neuroimage.2020.116531.

Natu V, O'Toole AJ. 2011. The neural processing of familiar and unfamiliar faces: A review and synopsis. British Journal of Psychology. doi:10.1111/j.20448295.2011.02053.x.

Nemrodov D, Niemeier M, Mok JNY, Nestor A. 2016. The time course of individual face recognition: A pattern analysis of ERP signals. NeuroImage. doi:10.1016/j.neuroimage.2016.03.006.

Nili H, Wingfield C, Walther A, Su L, Marslen-Wilson W, Kriegeskorte N. 2014. A Toolbox for Representational Similarity Analysis. PLoS Computational Biology. 
doi:10.1371/journal.pcbi.1003553.

Northoff G, Heinzel A, de Greck M, Bermpohl F, Dobrowolny H, Panksepp J. 2006. Selfreferential processing in our brain-A meta-analysis of imaging studies on the self. NeuroImage. 31(1):440-457. doi:10.1016/j.neuroimage.2005.12.002.

Oosterhof NN, Connolly AC, Haxby J V. 2016. CoSMoMVPA: Multi-Modal Multivariate Pattern Analysis of Neuroimaging Data in Matlab/GNU Octave. Frontiers in Neuroinformatics. doi:10.3389/fninf.2016.00027.

Pascalis O, de Schonen S, Morton J, Deruelle C, Fabre-Grenet M. 1995. Mother's face recognition by neonates: A replication and an extension. Infant Behavior and Development. doi:10.1016/0163-6383(95)90009-8.

Patel GH, Sestieri C, Corbetta M. 2019. The evolution of the temporoparietal junction and posterior superior temporal sulcus. Cortex. doi:10.1016/J.CORTEX.2019.01.026.

Pierce K. 2004. The brain response to personally familiar faces in autism: findings of fusiform activity and beyond. Brain. 127(12):2703-2716. doi:10.1093/brain/awh289.

Pruim RHR, Mennes M, van Rooij D, Llera A, Buitelaar JK, Beckmann CF. 2015. ICAAROMA: A robust ICA-based strategy for removing motion artifacts from fMRI data. NeuroImage. doi:10.1016/j.neuroimage.2015.02.064.

Ramon M, Gobbini MI. 2018. Familiarity matters: A review on prioritized processing of personally familiar faces. Visual Cognition. doi:10.1080/13506285.2017.1405134. Rorden C. 2011. fMRI Simulator.

Rossion B, Jacques C. 2012. The N170: Understanding the Time Course of Face Perception in the Human Brain. In: The Oxford Handbook of Event-Related Potential Components.

Serre T, Wolf L, Bileschi S, Riesenhuber M, Poggio T. 2007. Robust object recognition with cortex-like mechanisms. IEEE Transactions on Pattern Analysis and Machine Intelligence. doi:10.1109/TPAMI.2007.56.

Smith SM. 2002. Fast robust automated brain extraction. Human Brain Mapping. 17(3):143-155. doi:10.1002/hbm.10062.

Smith SM, Brady JM. 1997. SUSAN-A New Approach to Low Level Image Processing. International Journal of Computer Vision. doi:10.1023/A:1007963824710.

Steinberg C, Bröckelmann AK, Rehbein M, Dobel C, Junghöfer M. 2013. Rapid and highly resolving associative affective learning: Convergent electro- and magnetoencephalographic evidence from vision and audition. Biological Psychology. doi:10.1016/j.biopsycho.2012.02.009.

Sugiura M. 2014. Neuroimaging studies on recognition of personally familiar people. Frontiers in Bioscience. doi:10.2741/4235.

Vida MD, Nestor A, Plaut DC, Behrmann M. 2017. Spatiotemporal dynamics of similarity- 
based neural representations of facial identity. Proceedings of the National Academy of Sciences. doi:10.1073/pnas.1614763114.

Visconti Di Oleggio Castello M, Halchenko YO, Guntupalli JS, Gors JD, Gobbini MI. 2017. The neural representation of personally familiar and unfamiliar faces in the distributed system for face perception. Scientific Reports. doi:10.1038/s41598-017-12559-1. 